\title{
Introgression of Cajanus platycarpus genome into cultivated pigeonpea, C. cajan
}

\author{
Nalini Mallikarjuna*, Deepak Jadhav and Prabhakar Reddy ${ }^{1}$ \\ International Crops Research Institute for Semi Arid Tropics (ICRISAT), Patancheru 502 324, Andhra Pradesh, \\ India; ${ }^{1}$ Special project assistant till December, 2003 \\ (*author for correspondence: e-mail: N.Mallikarjuna@CGIAR.ORG)
}

Received 15 June 2005; accepted 22 November 2005

Key words: Cajanus platycarpus, Cajanus cajan, pigeonpea, interspecific hybridization, embryo rescue, diploid, tetraploid

\section{Summary}

Cajanus platycarpus, an incompatible wild species from the tertiary gene pool of pigeonpea (C. cajan (L.) Millspaugh), has many desirable characteristics for the improvement of cultivated varieties. To necessitate such transfers, embryo rescue techniques were used to obtain $F_{1}$ hybrids. The $F_{1}$ hybrids were treated with colchicine to obtain tetraploid hybrids, that were selfed to obtain $F_{2}, F_{3}$ and $F_{4}$ progenies. All of the hybrids and subsequent progenies had an intermediate morphology between the two parents. Backcrossing of the tetraploid hybrids with cultivated pigeonpea was not possible given embryo abortion, with smaller aborted embryos than those obtained in the $\mathrm{F}_{0}$ parental cross.

As a route of introgression, diploid $\mathrm{F}_{1}$ hybrids were backcrossed with cultivated pigeonpea and $\mathrm{BC}_{1}$ progeny obtained by in vitro culture of aborting embryos. $\mathrm{BC}_{2}$ plants were obtained by normal, mature seed germination. Although embryo rescue techniques had to be used to obtain $\mathrm{F}_{1}$ and $\mathrm{BC}_{1}$ plants, it was possible to produce $\mathrm{BC}_{2}$ and subsequent generations through direct mature seed. Every backcross to cultivated pigeonpea increased pollen fertility and the formation of mature seeds.

\section{Introduction}

Wild species of crop plants are placed in secondary or tertiary gene pools based on their crossability with cultivated species. Pigeonpea (Cajanus cajan (L.) Millspaugh.) has a rich gene pool in its various wild species. Many of the wild species from the secondary gene pool are compatible with cultivated pigeonpea and have been successfully used to transfer genes and traits of interest (Saxena et al., 1992; Saxena et al., 2000; Saxena \& Kumar, 2003; Mallikarjuna \& Saxena, 2005).

There are also many wild relatives of pigeonpea that are incompatible with cultivated species but have desirable characteristics that would improve pigeonpea as a crop (Saxena et al., 1996; Reddy et al., 1996). Among these, Cajanus platycarpus has received considerable attention because it has many desirable traits important for pigeonpea improvement. Some of these traits are extra-early flowering and maturity, photoperiod insensitivity, prolific flowering and pod setting, annuality, rapid seedling growth, salinity tolerance, and resistance to phytophthora blight, cyst nematode, and Helicoverpa (Dundas, 1985; Subbarao, 1988; Reddy et al., 1996; Mallikarjuna \& Moss, 1995). Cajanus platycarpus, although placed in the tertiary gene pool of pigeonpea, is now amenable to gene transfer with the development of suitable embryo rescue techniques (Mallikarjuna, 1998; 2003).

The major limitation in successfully using $C$. platycarpus for the improvement of cultivated pigeonpea is embryo abortion in the $\mathrm{BC}_{1}$ generation from the cross $C$. platycarpus $\times C$. cajan. Since embryo rescue is a time consuming technique in pigeonpea, our earlier attempts focused on treating diploid $(2 n=22)$ 
$\mathrm{F}_{1}$ hybrids with colchicine to double the chromosome number and obtain tetraploid $(2 n=44) \mathrm{F}_{1}$ hybrids (Mallikarjuna \& Moss, 1995). The progeny were selfed to obtain tetraploid $(2 n=44) \mathrm{F}_{2}$ plants. Selfing the $\mathrm{F}_{2}$ plants gave rise to mature seeds. Reciprocal crosses using $C$. cajan as the female parent and $C$. platycarpus as the pollen donor were not successful.

Unfortunately, the selfed progeny (tetraploid, $2 n=$ $44, \mathrm{~F}_{3}$ and $\mathrm{F}_{4}$ ) from the cross $C$. platycarpus $\times C$. cajan still had intermediate morphology with respect to growth habit, branching pattern, leaf and flower shape. In order to introduce more pigeonpea characters, $F_{1}$ (diploid, $2 n=22$ ) plants were backcrossed to cultivated pigeonpea.

The present study reports successful generation of backcross progeny by the use of in vitro techniques and conventional backcross program. The study also shows that it is possible to transfer important traits such as resistance to Phytophthora blight from C. platycarpus, although it is distantly related to cultivated pigeonpea.

\section{Materials and methods}

$\mathrm{F}_{1}$ hybrid plants $(C$. platycarpus $\times C$. cajan $)$ were obtained by rescuing aborting hybrid embryos in vitro (Mallikarjuna, 1998). Apical buds of $\mathrm{F}_{1}$ hybrids $(2 n=$ 22) were treated with an aqueous solution of $0.05 \%$ colchicine with $10 \%$ Tween-20 using a soaked cotton swab placed on the apical buds. After three days of colchicine treatment, apical buds were washed with water and allowed to grow. All the auxiliary buds and branches were excised. Hybrids were selfed to obtain tetraploid $\mathrm{F}_{2}(2 n=44)$ progeny.

Embryo rescue and tissue culture techniques were as described by Mallikarjuna and Moss (1995). $F_{1}$ hybrids were backcrossed to the cultivated parent $C$. cajan. All the $\mathrm{BC}_{1}(2 n=22)$ embryos aborted. To obtain $\mathrm{BC}_{1}$ plants, aborting ovules/immature seeds were rescued using the technique developed to save $\mathrm{F}_{1}$ hybrids. Aborting ovules/immature seeds from $\mathrm{BC}_{1}$ plants $(2 n=22)$ were supported on filter paper bridges and cultured on MS liquid medium with NAA $(0.5 \mathrm{mg}$ $\left.\mathrm{L}^{-1}\right)$ and BAP $\left(1.0 \mathrm{mg} \mathrm{L}^{-1}\right)$. After 3 weeks of ovule culture, embryos were dissected from the ovules and transferred to MS semi-solid medium with NAA $(0.1 \mathrm{mg}$ $\left.\mathrm{L}^{-1}\right)$ and BAP $\left(1.0 \mathrm{mg} \mathrm{L}^{-1}\right)$. Shoots which did not have a good root system were transferred to rooting medium which consisted of 1/10 MS basal medium with NAA (2 $\left.\mathrm{mg} \mathrm{L}^{-1}\right)$ and IBA $\left(1.0 \mathrm{mg} \mathrm{L}^{-1}\right)$. After 15-18 days on the rooting medium, shoots were transferred to 1/10 MS basal medium devoid of any growth regulators. Shoots with robust root system were transferred to soil, grown and maintained in the glasshouse. $\mathrm{BC}_{2}$ and $\mathrm{BC}_{3}$ plants were obtained by mature seed germination.

The pathogen Phytophthora drechsleri Tucvker f. sp. Cajani was isolated from small pieces of $3 \mathrm{~mm}$ stem portions having lesions of Phytophthora fungi growing on pigeonpea plant. The stem pieces were washed in running tap water and surface sterilized in $2 \%$ sodium hypochlorite solution for 1-3 minutes and placed on potato dextrose agar (PDA) slants. On the basis of growth characteristics, slants with the fungus in pure form were identified and confirmed by microscopic examination. The $\mathrm{P}_{3}$ isolate was confirmed by virulence test by inoculating 12-15 days old susceptible (ICPL 87119 , susceptible to $P_{2}$ and $P_{3}$ ) and resistant seedlings (ICP 2366, susceptible to $\mathrm{P}_{3}$ but resistant to $\mathrm{P}_{2}$ ) with the inoculum. All the susceptible seedlings were killed by $\mathrm{P}_{2}$ and $\mathrm{P}_{3}$ isolates but among the resistant seedlings, they were healthy against $\mathrm{P}_{2}$ isolate but succumbed to $\mathrm{P}_{3}$ isolate.

$\mathrm{F}_{2}$ seedlings with one trifoliate leaf ( $\leq 15$ days) were scored for the disease. The screening procedure was as follows: an inoculation concentration of $1 \mathrm{~g}$. of mycelium $/ 100 \mathrm{ml}$ of water, was sprayed on the seedlings. The seedlings were incubated at $25-30^{\circ} \mathrm{C}$ at 95-100\% humidity for 36 hours. Plants were sprayed with tap water every 2-3 hours during the day, until 4 days after inoculation. Disease data was taken after 10 days of inoculation. Plants which did not succumb to the disease were scored as resistant and the ones which succumbed to the disease were scored as susceptible. The screening procedure was as described by Gupta et al. $(1,8)$. After 30 days of sowing, the seedlings which did not succumb to the disease were again inoculated with Phytophthora pathogen and observations were recorded. Seedlings which showed resistance at the seedling stage were found to be resistant at 30 days too. Plants grew normally and set seeds.

Immature flower buds from $F_{1}$ diploid and $F_{1}$ tetraploid plants were fixed in Carnoy's II mixture (alcohol: acetic acid: chloroform; 6:3:1) at $4{ }^{\circ} \mathrm{C}$ for meiotic analysis. After 24 hours in Carnoys's II, buds were transferred to Carnoy's I (alcohol: acetic acid; $3: 1$ ). Buds were squashed and stained in $2 \%$ acetocarmine and meiotic analyses were made on suitable preparations. Pollen fertility analysis was conducted by staining the pollen grains in $2 \%$ aceto carmine solution. Pollen grains staining bright pink were counted as fertile grains and stainless grains were counted as sterile. 
Chi - square $\left(\chi^{2}\right)$ analysis: Chi - square test $\left(\chi^{2}\right)$ to find the goodness of fit was calculated as per the formula using the genstat version 6.1(Payne, 2002).

$$
\begin{aligned}
& \chi^{2}= \\
& \Sigma \frac{(\text { Observed frequencies }- \text { Expected frequencies })^{2}}{\text { Expected frequencies }}
\end{aligned}
$$

\section{Results and discussion}

Diploid $\mathrm{F}_{1}$ hybrids from the cross $C$. platycarpus $\times C$. cajan were obtained as a result of rescuing aborting hybrid embryos in vitro (Figure 1-6, 1-7, 1-8, 1-9). It took 6-8 months from embryo rescue to transfer of $F_{1}$ hybrid plants to soil. The $\mathrm{F}_{1}$ hybrids had intermediate morphology, with semi-trailing branches. (Figure 4-2). The floral axis resembled that of $C$. cajan. In order to introduce more cultivated pigeonpea characteristics, $\mathrm{F}_{1}$ hybrids were backcrossed with cultivated pigeonpea; however, embryos aborted in the $\mathrm{BC}_{1}$ cross. In order to avoid embryo rescue for the second time, $\mathrm{F}_{1}$ hybrids $(2 n=22)$ were treated with colchicine to double their chromosome number. The success of doubling the chromosome number in the $\mathrm{F}_{1}$ hybrids was $2 \%$.

Tetraploid $F_{1}$ plants $(2 n=44)$ had robust growth with intermediate morphology between the two parents with delicate branches and semi-trailing habit (Figure 1-3), and produced a large number of mature seeds. Tetraploid $\mathrm{F}_{2}$ plants had robust growth with intermediate morphology between the two parents. There was not much difference in morphology between $\mathrm{F}_{2}$ and $\mathrm{F}_{3}$ hybrids (Figure 1-4, 1-5), and $\mathrm{F}_{2}$ seeds were obtained in large numbers. The seeds had the shape of cultivated pigeonpea (Figure 3-5, 3-7) but with prominent strophiole and were black in color as in the wild species (Figure 3-6). In the $\mathrm{F}_{3}(2 n=44)$, seeds were semiblack in color with a few tan colored seeds, and the size of the strophiole had reduced (Figure 3-8). In the field the tetraploid F3 plants because of their semi-trailing growth habit and large leaves covered the ground.

In order to check if genes from $C$. platycarpus introgressed into cultivated pigeonpea, a small random sample of $60 \mathrm{~F}_{2}$ seeds were germinated in vivo and the seedlings were screened for Phytophthora blight disease, as $C$. platycarpus is the only wild species with reported resistance to the disease (Reddy at al., 1996). The progeny segregated into 1R:3S the expected Mendelian segregation $\left(\chi^{2}=0.02 ; p=0.88\right)$. A random sample of $46 F_{2}$ derived $F_{3}$ seedlings were screened for Phytophthora blight, with 9 seedlings
Table 1. Seed set in the cross Cajanus platycarpus ICPW $69 \times C$. cajan ICPL 85010.

\begin{tabular}{lclll}
\hline & $\mathrm{F}_{1}$ & $\mathrm{BC}_{1}$ & $\mathrm{BC}_{2}$ & $\mathrm{BC}_{3}$ \\
\hline $\begin{array}{l}\text { No. pollinations } \\
\text { No. pod set (\%) }\end{array}$ & 1183 & 788 & 901 & 506 \\
$\begin{array}{l}\text { No. immature } \\
\quad \text { pods (\%) }\end{array}$ & $259(22)$ & $220(28)$ & $207(23)$ & $242(48)$ \\
$\begin{array}{l}\text { No. mature } \\
\quad \text { pods (\%) }\end{array}$ & 0 & $209(27)$ & $85(9)$ & $45(9)$ \\
$\%$ pollen fertility & 8 & $11(1)$ & $122(14)$ & $197(39)$ \\
\end{tabular}

resistant and 37 seedlings susceptible. The Chi-square values fitted into the ration of 1:3 and was significant at $5 \%(<0.05)$. Selfing the plants to produce $\mathrm{F}_{3}$ (Figure 3-8) and $\mathrm{F}_{4}$ seeds were effortless and there was little change in plant and seed morphology. It was not possible to backcross any of the tetraploid progeny $\left(\mathrm{F}_{1}, \mathrm{~F}_{2}\right.$, $\mathrm{F}_{3}$ or $\mathrm{F}_{4}$ ) with cultivated pigeonpea to introduce more pigeonpea characteristics, as hybrid embryos were abnormal and aborted much earlier than in the $\mathrm{F}_{0}$ cross. They did not respond to embryo rescue techniques due to their smaller size.

The diploid $F_{1}$ plants were backcrossed to cultivated parent. Embryos aborted between 15-20 days after pollination as seen in $\mathrm{F}_{0}$ parental crosses, but the size of the aborting embryo was bigger than in the $\mathrm{F}_{0}$ cross, being $0.25-0.5 \mathrm{~mm}$ in size, and at the cotyledonary stage of development. Embryos grew and formed plants on the embryo rescue medium developed for embryos from $C$. platycarpus $\times C$. cajan cross.

The diploid $\mathrm{BC}_{1}$ hybrid plant had intermediate growth habit with semi-trailing branches. Morphologically, there were no differences between $\mathrm{F}_{1}$ and $\mathrm{BC}_{1}$ plants. A small number (1\%) of mature and a large number (27\%) of immature seeds were obtained on the $\mathrm{BC}_{1}$ plants (Table 1). Only the mature seeds were used to develop $\mathrm{BC}_{2}$ plants.

$\mathrm{BC}_{2}$ plants had more of cultivated pigeonpea characteristics, with erect growth habit (Figure 4-3). Flowers were tripped to encourage the development of seeds, but seed set from self pollinations was not observed in spite of increased pollen fertility of $38 \%$ (Table 1). $\mathrm{BC}_{2}$ plants were backcrossed to cultivated pigeonpea and a large number of seeds were obtained. With the further introduction of the cultivated pigeonpea genome $\mathrm{BC}_{3}$ diploid plants (Figure 4-4) resembled the cultivated parent that was also the pollen parent (Figure 4-5, 4-6).

$\mathrm{F}_{1}$ hybrid pods $(2 n=22)$ morphologically resembled the female parent $C$. platycarpus pods 

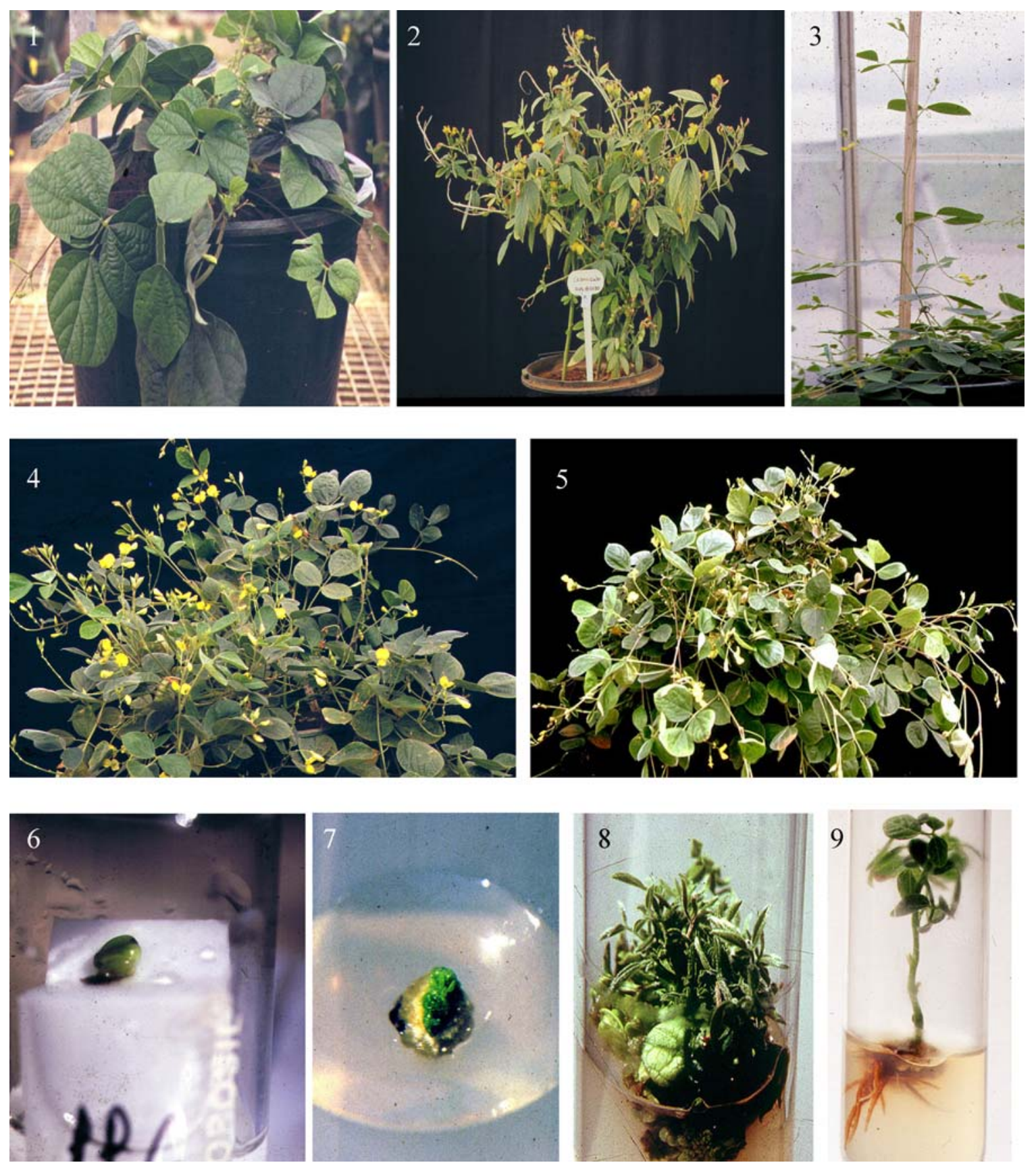

Figure 1. (1) Female parent, wild species Cajanus platycarpus. (2) Male parent, cultivated pigeonpea Cajanus cajan. (3) $\mathrm{F}_{1}$ (tetraploid) hybrid between $C$. platycarpus $\times C$. cajan. (4) $\mathrm{F}_{2}$ (tetraploid) hybrid between $C$. platycarpus $\times C$. cajan. (5) $\mathrm{F}_{3}$ (tetraploid) hybrid between $C$. platycarpus $\times$ C. cajan. (6) In-ovulo embryo culture to save aborting hybrid embryos. (7) Hybrid embryo culture. (8) Multiple shoots from hybrid embryo. (9) In vitro rooting of hybrid shoots. 

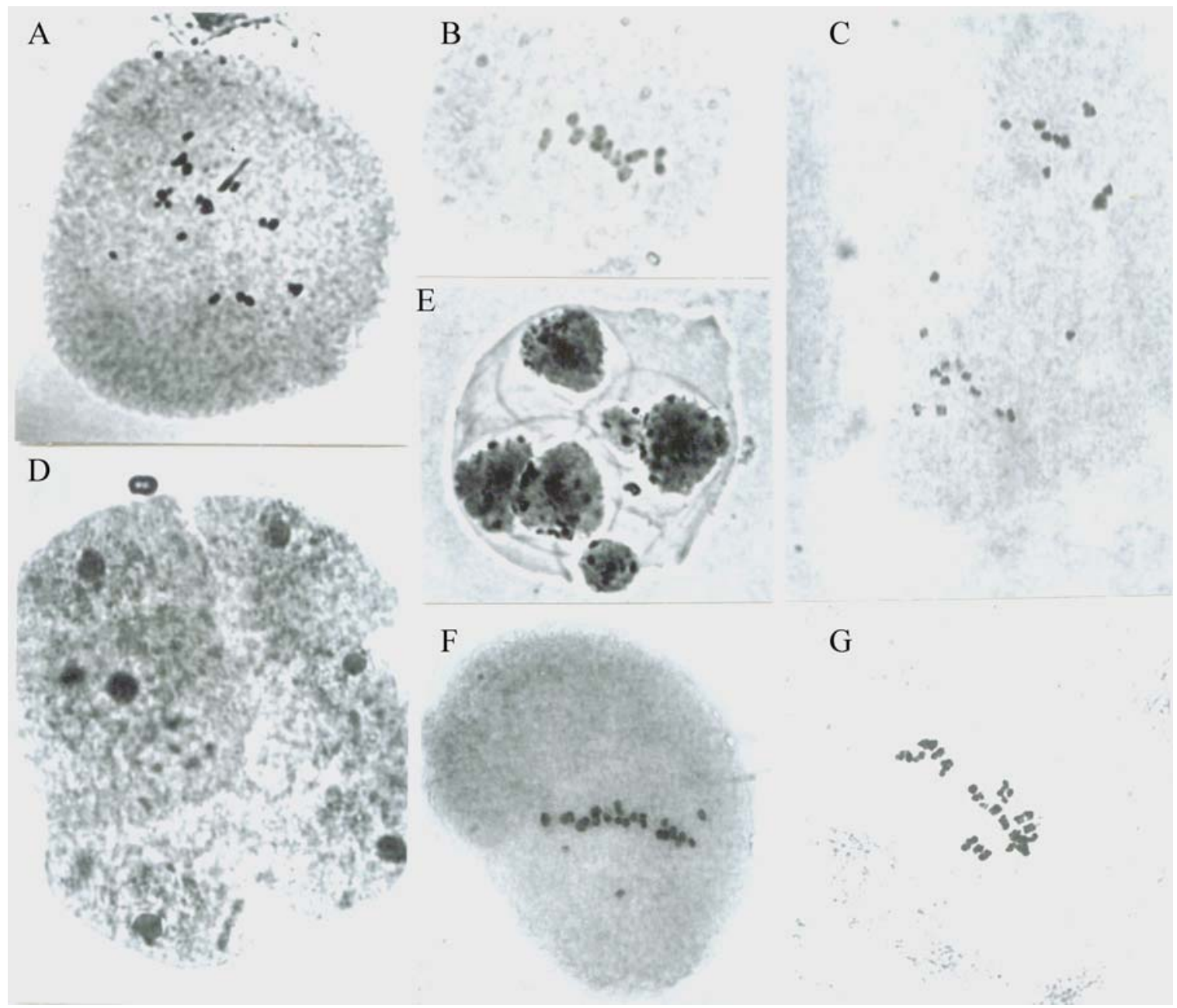

Figure 2. Meiotic analysis in the cross C. platycarpus $\times$ C. cajan: A-E $\mathrm{F}_{1}$ diploid; A $-\mathrm{F}_{1}$ hybrid showing 6 univalents. $\mathrm{B}-\mathrm{F}_{1}$ hybrid showing 12 bivalents, which is rarely seen. $\mathrm{C}-\mathrm{F}_{1}$ anaphase separation showing chromosomes not reaching the poles. $\mathrm{D}-\mathrm{F}_{1}$ telophase showing abnormal micronuclei in the tetrad. $\mathrm{F}-\mathrm{F}_{1}$ (tetraploid) metaphase showing the formation of univalents. $\mathrm{G}-\mathrm{F}_{2}$ (tetraploid) normal chromosome configuration in metaphase.

(Figure 3-1). $F_{2}$ pods resembled more the cultivated parent. Morphologically, there were no differences between $\mathrm{F}_{2}$ (Figure 3-2) and $\mathrm{F}_{3}$ pods (Figure 3-3). The $\mathrm{BC}_{1}, \mathrm{BC}_{2}$ and $\mathrm{BC}_{3}$ pods resembled the female parent (Figure 3-4).

Meiotic analyses of the $F_{1}$ diploid hybrid showed much variation in chromosome configurations with a mean of 6 univalents (ranging from 5-8) and 8 bivalents ranging from 7-9, trivalents and tetravalents were not observed. $F_{1}$ tetraploid hybrid had predominantly bivalent formation ranging from $10-14$, with a mean of 10 per cell and a mean of 6 tetravalents per cell. Univalents were rarely seen and trivalents were absent. $\mathrm{BC}_{1}$ plants showed a 1.5 univalents per cell ranging
Table 2. Chromosome association (per cell*) in $\mathrm{F}_{1}$ diploid, $\mathrm{F}_{1}$ tetraploid and $\mathrm{BC}_{1}$ hybrids.

\begin{tabular}{lllll}
\hline Hybrid & Univalents & Bivalents & Trivalents & Tetravalents \\
\hline $\mathrm{F}_{1}$ diploid & 6.0 & 8.0 & 0.0 & 0.0 \\
$\mathrm{~F}_{1}$ tetraploid & 0.0 & 10 & 0.0 & 6.0 \\
$\mathrm{BC}_{1}$ diploid** & 1.5 & 9 & 0.4 & 0.4 \\
\hline
\end{tabular}

${ }^{*}$ mean of 50 cells; ${ }^{* *}$ mean of 44 cells.

from 1-2, with a mean of 9 bivalents, trivalents and tetravalents were rarely seen (Table 2; Figure 2).

The number of mature and immature seed set varied according to the generation, suggesting a relationship 

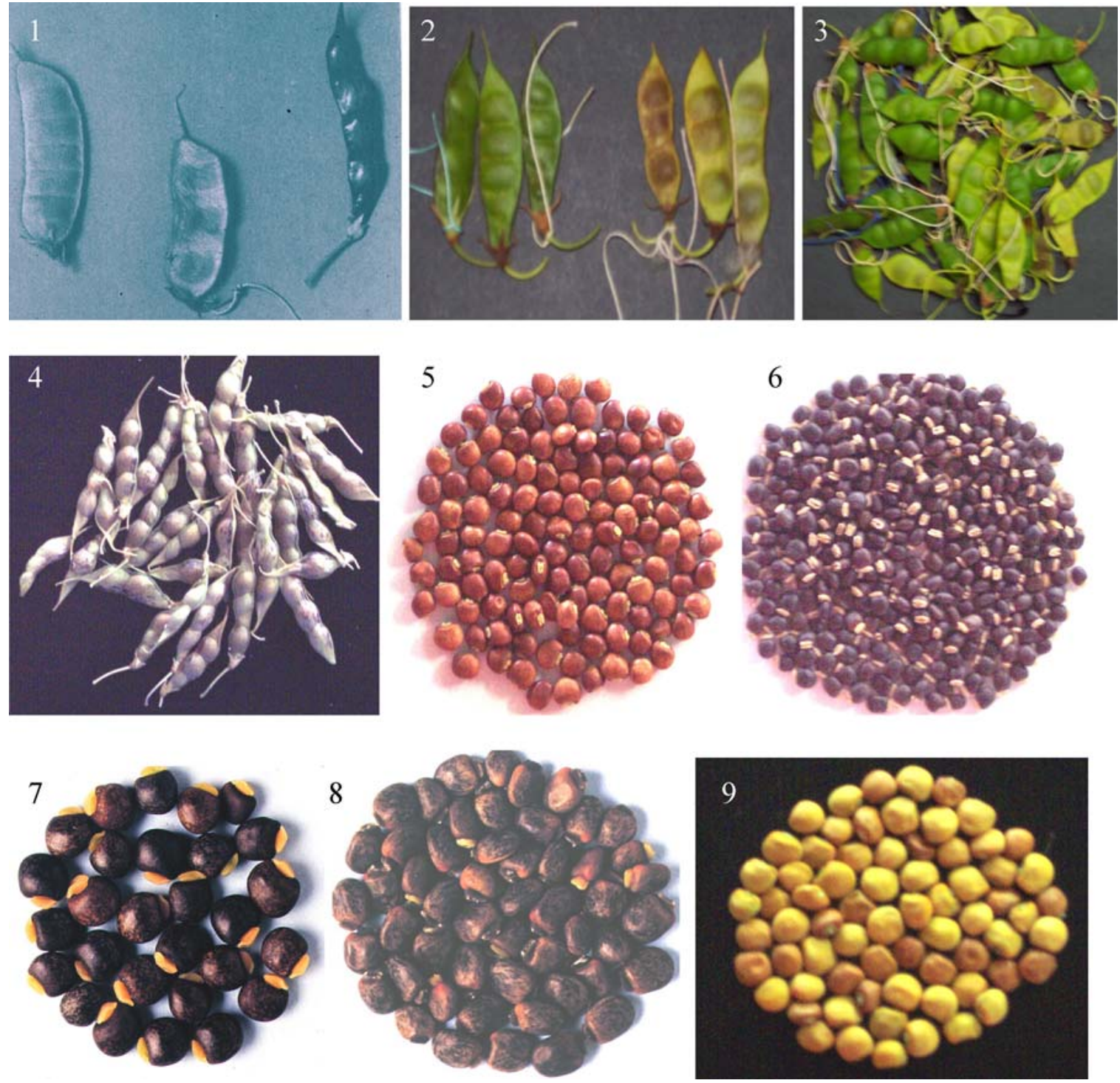

Figure 3. (1) Pod morphology of the two parents (left- female parent; right - male parent) and the hybrid at the center. (2) $\mathrm{F}_{2}$ (tetraploid) pods. (3) $\mathrm{F}_{3}$ (tetraploid) pods. (4) $\mathrm{F}_{1} \mathrm{BC}_{2}$ (diploid) pods. (5) Seeds of male parent, the cultivated species Cajanus cajan. (6) Seeds of female parent, the wild species Cajanus platycarpus. (7) Tetraploid $\mathrm{F}_{2}$ seeds. (8) Tetraploid $\mathrm{F}_{3}$ seeds. (9) Diploid $\mathrm{F}_{1} \mathrm{BC}_{2}$ seeds.

to the amount of cultivated genome present. In the $\mathrm{F}_{1}$ hybrid, which theoretically had $50 \%$ of wild species and $50 \%$ of cultivated genome, all pollinations had to be assisted by a growth regulator and all the seeds were immature. In the $\mathrm{BC}_{1}$ hybrid with theoretically $75 \%$ of cultivated genome and $25 \%$ of the wild genome, there was improvement in pollen fertility from $8 \%$ in $\mathrm{F}_{1}$ to $26 \%$, but the number of immature seeds far exceeded the number of mature seeds (Table 1). In the $\mathrm{BC}_{2}$, pollen fertility increased to $38 \%$ and more than $10 \%$ of the seeds were mature. It was not until the $\mathrm{BC}_{3}$ generation that it was possible to obtain more than $35 \%$ mature seeds (Figure 3-9).

When using wild species from the tertiary gene pool, it is usually necessary to use embryo rescue techniques at least once to obtain hybrid plants. Such a requirement has been observed in groundnut and chickpea wide crosses (Mallikarjuna \& Sastri, 2002, Mallikarjuna, 2003). In the present investigation, embryo rescue techniques had to be used more than once, 
but by doing so it was possible to transfer more pigeonpea characters into the hybrid that were clearly evident in the resulting morphology. Theoretically in each backcross generation, $50 \%$ of the genome of the hybrid plant was replaced by the genome of cultivated species. It took three backcrosses $\left(\mathrm{BC}_{3}\right)$ to the cultivated pigeonpea to incorporate the morphology of cultivated pigeonpea in the hybrid plants.

This is the first report in pigeonpea where an incompatible wild species from tertiary gene pool such as $C$. platycarpus has been successfully crossed with cultivated pigeonpea and fertile hybrids and backcross progeny obtained. Efforts are being made to obtain seeds in large number so that the progeny can be used to screen for various biotic and abiotic constraints. In rice, resistance to brown plant-hopper, white-backed plant-hopper and bacterial blight was transferred from wild species using embryo rescue techniques thrice to obtain $\mathrm{F}_{1}, \mathrm{BC}_{1}$ and $\mathrm{BC}_{2}$ hybrids (Brar \& Khush, 1997). But the source of resistance transferred in 1978 to bacterial blight from the wild species is being used even today. Hopefully, with the success in pigeonpea $\times C$. platycarpus crosses, similar transfers of enhanced resistance can be achieved.

The tetraploid hybrid plants form good ground cover, and hence can be used a forage plant and can be used as a graze for animals.

\section{Acknowledgements}

The authors thank the AP-Netherlands Biotechnology Project for funds to carry out the research.

\section{References}

Brar, D.S. \& G.S. Khush, 1997. Alien introgression in rice. Plant molecular biology 35: 35-47.
Dundas, I.S., 1996. Studies into overcoming some crossability barriers involving pigeonpea (Cajanus cajan (L.) Millsp.) and related Atylosia species. Project report 1984-86. International Crops research Institute for Semi Arid Tropics, Patancheru 502 324, India.

Mallikarjuna Nalini \& J.P. Moss, 1995. Production of hyrids between Cajanus platycarpus and C. cajan. Euphytica 83: 43-46.

Mallikarjuna Nalini, 1998. Ovule culture to rescue aborting embryos from pigeonpea (Cajanus cajan L. Millspaugh) wide crosses. Indian Journal of Experimental Biology 36: 225-228.

Mallikarjuna Nalini \& D.C. Sastri. 2002. Morphological, cytological and disease resistance studies of the intersectional hybrids between Arachis hypogaea L. and A. glabrata Benth. Euphytica 126(2): 161-167.

Mallikarjuna Nalini, 2003. Wide hybridization in important food legumes. In: P.K. Jaiwal and R.P. Singh (Eds.) Improvement Strategies of Leguminosae Biotechnology, pages 155-170. Kluwer Acad. Publishers.

Mallikarjuna Nalini \& K.B. Saxena, 2005. A cytoplasmic malesterility system derived from pigeonpea (Cajanus cajan (L) Millsp.) cytoplasm. Euphytica 142(1-2): 143-148.

Payne, R.W. 2002. The Guide to Gen Stat (R) Release 6.1. Part 2: Statistics. VSN International Ltd., Oxford. UK

Reddy, M.V., T.N. Raju \& V.K. Sheila, 1996. Phytophthora blight disease in wild pigeonpea. International Chickpea and Pigeonpea Newsletter 3: 52-53.

Saxena, K.B., R.P. Ariyanayagam \& L.J. Reddy, 1992. Genetics of a high selfing trait in pigeonpea. Euphytica 59: 125-127.

Saxena, K.B., M.V. Reddy, V.R. Bhagwat \& S.B. Sharma, 1996. Preliminary studies on the incidence of major diseases and insects in Cajanus platycarpus germplasm at ICRISAT Asia center. International Chickpea and Pigeonpea Newsletter 3: 51-52.

Saxena, K.B., R.V. Kumar \& P.V. Rao, 2000. Pigeonpea nutrition and its improvement. Genetic Resources and Enhancement Program, International Crops Research Institute for Semi Arid Tropics, Patancheru 502 324, Andhra Pradesh, India.

Saxena, K.B. \& R.V. Kumar, 2003. Development of a cytoplasmic-nuclear male-sterility system in pigeonpea using $C$. scarabaeoides (L.) Thours. Indian J Genet 63(3): 225-229.

Subbarao G.V., 1988. Salanity tolerance in pigeonpea (Cajanus cajan) and its wild relatives. Ph.D. Dissertation, Indian Institute of Technology, Kharagpur, India.

Subbarao, G.V., 1988. Salinity tolerance in pigeonpea (Cajanus cajan) and its wild relatives. Ph.D., dissertation, Indian Institute of Technology, Kharagpur, India. 